\title{
Providing Access to Rural Patients - A Management Decision
}

\author{
Kambiz Farahmand ${ }^{1}$, Mahmoud Mostafa ${ }^{2}$, Satpal Singh Wadhwa ${ }^{3}$,Sudhindra Upadhyaya ${ }^{4}$, \\ Vahid H Khiabani ${ }^{5}$
}

Abstract: The purpose of this study is to model the arrival of patients to a level one surgical department in a large city for two large departments namely orthopedic and Podiatry, and the cost associated with the arrivals for the Podiatry patients. Podiatry patients were chosen for the cost modeling as to set the ground worker for analyzing the feasibility of increased remote capability specifically in thePodiatry department within the referral area to minimize travel time and cost for the patients and the provider

\section{INTRODUCTION}

The geographic rural area under consideration includes New Mexico and West Texas which is mainly served by the surgical department located in Albuquerque New Mexico. The focus of this study is on the surgical referrals to this facility from three main areas including West Texas, North Albuquerque and South Albuquerque.

Patients who live in Southern or Northern New Mexico and West Texas who require non-emergency surgery are referred to the Albuquerque Medical Center surgical department, however, all emergency surgeries are referred to the nearest clinic or hospital with surgical capability.

Two departments which have significant non-emergency surgical referrals from outside Albuquerque include Orthopedic and Podiatry surgeries. The referral patients will also have pre and post-surgery visits to the Albuquerque Medical center. Considering the volume of referral patients for these departments and the cost associated with the referrals, it may be feasible to build additional clinics or Medical centers with pre or post or surgical capability in areas such as West Texas or other locations in New Mexico.

\section{LITERATURE REVIEW}

One of the educational and clinical goal and objectives of the integrated health care program was to improving the health of Veterans by integrating mental and behavioral health services into Patient Aligned Care Teams (PACTs), where Veterans receive their primary care. This integration requires significant transformation of the way that mental health professionals provide care. Evidence-based innovations in brief assessments and treatments are needed for the primary care population.

Creemers, Lambrecht, and Vandaele described the healthcare sector as a fast growing segment of GNP in almost every economy. No wonder that we witnessed a tremendous increase in research both medical research to improve medical practice but also research to improve management practices. Patient flow management is an example. Patient flow represents the ability of the healthcare system to serve patients quickly, reliably and efficiently as they move through stages of care. Unfortunately, patients still experience delays and waiting lists. A queuing model offers an excellent tool to analyze and to improve the performance of healthcare systems. The purpose of the contribution was to discuss differences with the modeling of manufacturing systems and to focus on modeling issues in patient flow. Next, they discuss two specific topics: first, the impact of interrupts and absences on waiting lists and delays, and second, the modeling of batches in healthcare operations.

Meijboom, Schmidt-Bakx, and Westert discussed organizational problems that occur in situations that are complex because the treatment of patients requires input from multiple health care providers, and to argue conceptually how to resolve these problems by using SCM practices. First SCM, being related to settings where several companies contribute to the production of one particular product, was discussed in general. Since patient care is about service provision, the next to be examined was service supply chains. Subsequently, major challenges in patient-oriented care provision followed in settings where several health care providers are involved, based on which opportunities for applying SCM in patient care were presented. Based on literature addressing country comparisons of patient

${ }^{1}$ Corresponding Author: Kambiz.Farahmand@ndsu.edu 


\section{American Research Journal of Medicine and Surgery, Volume 1, Issue 1, 2015}

ISSN 2379-8955

experiences, four major problem categories are distinguished: communication, patient safety, waiting times, and integration. Although problems also occur within organizational boundaries, the steps from one provider to the next generally represent the weakest spots in a system of health care providers. By applying insights from SCM, these problems can be tackled. Problems with communication and integration might well benefit from the nomination of care coordinators. Information gathering and processing, i.e. both the availability of medical records of individual patients and information on provider performance, has to be improved. Breaking down functional barriers between care-silos, within health care providers as well as inter-organizationally, is a necessary condition for enhanced patient-centered integration. Policy should also stimulate the provision of more coordinated services, for example, through integral cost prices for separate diseases ("case-mixed accounting").

Kuo, Leung, and Graham were motivated by the resource allocation problem in the Emergency Department at the Prince of Wales Hospital in Hong Kong. They adopted a simulation approach to analyze how the allocation decisions influences patient's experience in the department. The development of the model was complicated by the fact that there were different categories of patients (with different time-varying arrival rates, treatments and procedures), and the data records were incomplete to allow direct estimation of many of the key operational parameters (e.g. the duration of doctor's consultation). To tackle the first issue, patients' arrivals were modeled as Poisson processes with category and time-dependent arrival rates. The second issue was resolved by positing a general distribution (Weibull) for some key processes, and developing meta-heuristic approaches to jointly estimate the distribution parameters. Their computational results showed that accurate estimates of the distribution parameters were found using the authors' proposed search procedure, in that the simulated results and the actual data were consistent.

Bowers explained that Patients' experiences of waiting for treatment have changed dramatically in recent years in the United Kingdom's National Health Service. There has been a substantial reduction in the mean wait but the characteristics of the distributions have also changed significantly, implying a change in priorities and waiting list management. Simulations are often used to assess proposals for investment and reorganization that might affect waiting time. However, a more realistic model incorporating waiting list management behavior was needed since empirical distributions of waiting times indicate that a first-in-first-out model was not valid. This study developed a model of waiting list management that included an explicit measure of priority associated with the patient's wait compared to a specified target. The model can generate a range of behaviors, from the near negative exponential distributions associated with some historic examples of waiting list management, through to the well-defined modes of a rigid first-in-first-out system. In one illustration of the use of the waiting list management model, the impact of the seasonal variations in demand and supply was explored. Simulation experiments demonstrated the consequent seasonality in waiting times, implying a need for care when monitoring progress towards any targets.

Yang, Sullivan, Wang, and Naidu mentioned that in the current health care service, it is important to minimize staff idle time and maintain a high utilization rate of the medical facilities. More and more hospitals, however, recognize waiting is a universal and serious problem for patients. The critical problem in medical scheduling is how to keep the balance of all these factors. Computer simulation is a powerful tool for medical management and scheduling. This paper presents a literature review of the current state of computer simulation in medical scheduling. Various approaches to simulate the operations in medical area, as well as analysis of these approaches along with their effectiveness are presented in this paper. They anticipated such a survey would help them to develop a computer simulation system for use at Duke University's medical oncology department.

Niu and et. el. Mentioned that the operating room (OR) is one of the most demanding departments in Winnipeg Health Science Center (WHSC). The problems faced to the OR are the long waiting list of patients and the inefficient utilization of human resources and facilities. The OR needs to treat a large variety of patient types versatility and dynamically. A discrete event simulation tool is used for modeling the OR operation. The major work involving in this research includes data collection, simulation modeling, model validation, and output analysis. The initial results have shown the simulation potential in the performance improvement of healthcare systems.

Knotterus, Joosten, and Daams examined the quality of referrals of four general practitioners, two with high and two with average rates of referral to the department of internal medicine was judged by an independent expert panel. The panel, consisting of two general practitioners and one specialist, reviewed a set of information about the referrals blindly and random sequence. The same distribution of quality of referrals was found among the referrals of the two high referring general practitioners $(n=192)$ as among those of the general practitioners with average rates $(n=88)$; that is, $57 \%$ and $55 \%$ respectively, of the cases had clear medical indications for referral, while the data did not permit a conclusion in $15 \%$ and $10 \%$, respectively, of the cases. Controlling for sex, age, and status of the referral 


\section{American Research Journal Of Medicine And Surgery, Volume 1, Issue 1, 2015}

ISSN 2379-8955

(first or repeat referral) did not alter the results. They concluded that using the referral rates to judge referral quality is misleading. However, a blind and randomly performed panel review of referrals is a time consuming but feasible method of quality assessment.

El-Darzi, Vasilakis, Chaussalet, and Millard studied the flow of patients through geriatric hospitals has been previously described in terms of acute(short-stay), rehabilitation(medium-stay), and long-stay states where the bed occupancy at a census point is modeled by a mixed exponential model using BOMPS(Bed Occupancy Modeling and Planning System). In this, a patient is initially admitted to acute care. The majority of the patients are discharged within a few days into their own homes or through death. The rest are converted into medium-stay patients where they could stay for a few months and there after either leave the system or move onto along-stay compartment where they could stay until they die. The model forecasts the average length of stay as well as the average number of patients in each state. The average length of stay in the acute compartment is artificially high if some would-be longterm patients are kept waiting in the short-stay compartment until beds become available in long-stay (residential and nursing homes). In this paper, the authors considered the problem as a queuing system to assess the effect of blockage on the flow of patients in geriatric departments. What-if analysis is used to allow a greater understanding of bed requirements and effective utilization of resources.

Van Berkel and Blake described the use of operational research techniques to analyze the waitlist for the Division of General Surgery at the Capital District Health Authority in Halifax, Nova Scotia, Canada. A discrete event simulation model was developed to aid capacity-planning decisions and to analyze the performance of the division. The analysis examined the consequences of redistributing beds between sites, and achieving standard patient lengths of stay, while contrasting them to current and additional resource options. From the results, multiple independent and combined options for stabilizing and decreasing wait times for elective procedures were proposed.

Duguay Chetouane described a discrete event simulation study of an emergency department at Dr. Georges-L. Dumont Hospital in Moncton (Canada). The objective of the study was to reduce patient waiting times and to improve overall service delivery and system throughput. As patient waiting times are linked to resource availability, a number of alternatives were designed based on adding resource scenarios. Model development was made using Arena software.

Schofield et. al. established the rate of and reasons for cancellations of surgery on the scheduled day in a major metropolitan Australian tertiary hospital using a prospective survey. The main outcome measures were: proportion of operations cancelled on the day of surgery, obtained each day from the operating theatre list and a separate list of additions and cancellations compiled on the day; reasons for cancellations from the cancellation list, extended or confirmed, as necessary, by questioning of bookings and ward staff, or members of the surgical team; and estimated and actual duration of each operation and patient information from hospital clinical records. The results were 133 surgeons scheduled 7913 theatre sessions in the study period; 941 of these $(11.9 \%)$ were cancelled on the day, including 724 of $5472(13.2 \%)$ elective procedures on working weekdays. Main reasons for cancellation were no theatre time due to over-run of previous surgery (18.7\%); no postoperative bed (18.1\%); cancelled by patient (17.5\%); and change in patient clinical status (17.1\%). Procedural reasons (including patient not ready, no surgeon, list error, administrative cause, and communication failure) totaled $21.0 \%$. Ear, nose, and throat surgery experienced the most cancellations (19.6\%), followed by cardiothoracic surgery (15.8\%). There were five major reasons of similar magnitude for on-the-day surgery cancellations. The authors estimated that $60 \%$ of cancellations of elective procedures were potentially avoidable. Change of one factor leading to cancellation (e.g. provision of more postoperative beds) is not likely to lead to improvement unless the other major factors are also tackled.

\section{Clustering TeChNiQues}

Radial clusters are formed around the Albuquerque medical center to allow for utilization of average distances from each cluster to the medical center for simulation purposes.

Clustering is the organization of patterns into groups based on similarity. Intuitively, patterns within each cluster are more similar to each other than they are to a pattern belonging to a different cluster. Let $\mathrm{X} \in \mathrm{R}^{\mathrm{m} \times \mathrm{n}}$ a set of observations representing a set of $\mathrm{m}$ points $x_{i}$ in $\mathrm{R}^{\mathrm{n}}$. The goal is to partition $\mathrm{X}$ into $\mathrm{K}$ groups $\mathrm{C}_{\mathrm{k}}$ where every data that belong to the same group are more alike than data in different groups. Each of the groups is called a cluster. The result of this algorithm is a mapping of data items $x_{i}$ to clusters $\mathrm{C}_{\mathrm{k}}$. The number $\mathrm{K}$ might be pre-assigned by the user or it can be predetermined by the user, Fung. G (2001).

Clustering is useful in several exploratory pattern-analysis, grouping, decision-making, image segmentation, and pattern classification. However, in many situations, there is little prior information available about the data, and the 


\section{American Research Journal of Medicine and Surgery, Volume 1, Issue 1, 2015 ISSN 2379-8955}

decisions must be made with very few assumptions. It is under these conditions that clustering technique is particularly useful for exploring inter relationships among the data points to make an assessment (perhaps preliminary) regarding their structure, Jain, Murty \& Flynn (1999).

Clustering is mainly based on two major techniques: 1) hierarchical, 2) partitional as shown in Figure 1. A partition based clustering is division of the dataset into non-overlapping subsets (clusters) such that each observation in the dataset is in exactly one subset. In hierarchical clustering technique, clusters have sub clusters, which is a set of nested clusters that are organized as a tree. Each node (cluster) in the tree (except for the leaf nodes) is the union of its children (sub clusters), and the root of the tree is the cluster containing all the objects, Pang-Ning, Steinbach \& Kumar (2006).

Consider a set of $\mathrm{n}$ observations, $x_{1, \ldots \ldots} x_{n}$ where each observations $\mathrm{x}_{\mathrm{i}}$ is a vector in $\mathrm{R}^{\mathrm{d}}$, the $\mathrm{k}$ means algorithm aims at partitioning the $\mathrm{n}$ observations into $\mathrm{k}(\mathrm{k} \leq \mathrm{n})$ sets of clusters, $\mathrm{S}_{1}, \mathrm{~S}_{2} \ldots \mathrm{S}_{\mathrm{k}}$ so as to minimize the following function $\mathrm{D}=\sum_{i=1}^{n} \sum_{j=1}^{k} r_{i j}\left\|x_{i}-c_{j}\right\|$, here $r_{i j}=1$ if the observation $x_{i}$ is assigned to the set of $S_{j}$ and $r_{i j}=0$ otherwise, and $c_{j}$ is the centroid of the observations within the set $S_{j . .}$ Starting with a random set of k centroids $\mathrm{c}_{1}(0) \ldots \ldots . . \mathrm{c}_{\mathrm{k}}(0) \in \mathrm{R}^{\mathrm{d}}$ the algorithm alternates an assignment and a refinement step. During the assignment step, each observation $x_{i}$ is assigned to the set characterized by the nearest centroid, Pang-Ning, Steinbach, \& Kumar (2006).

$$
r_{i j}=\left\{\begin{array}{rr}
1 & \text { if } h=\operatorname{argmin}_{j}\left\|x_{i}-c_{j}(t)\right\| \\
0 & \text { else }
\end{array}\right.
$$

During the refinement step each centroid is updated based on the equation

$$
c_{j}(T+1)=\frac{\sum_{I=1}^{n} r_{i j}(T) x_{i}}{\sum_{l=1}^{n} r_{i j}(T)}
$$

In this study, patients, and the cities they come from, are clustered based on the distance they travel to reach Albuquerque in order to simplify the cost calculations. The number of partitions was arbitrarily chosen to be five. The means for these partitions are shown in Table 1 and they are labeled in Figure 1. Cluster 5 is the closest cluster to Albuquerque, covering the city and a small area around it.

Table 1: The five clusters and their average distance to the medical center

\begin{tabular}{|c|c|c|}
\hline Cluster & Average one way Distance to ALBQ in miles & Color Code \\
\hline CL1 & 611.5319149 & Dark Red \\
\hline CL2 & 882.609375 & Light Green \\
\hline CL3 & 415.114159 & Green \\
\hline CL4 & 193.2776745 & Yellow \\
\hline CL5 & 25.18008418 & Brown \\
\hline
\end{tabular}

Figure 1 shows the five clusters formed in the region of New Mexico and West Texas served by the medical center and their radial configuration as we move away from Albuquerque. The average hourly arrival rate for each cluster used to simulate the arrival of patients from each zone in the geographic region under consideration is shown in Table 2.

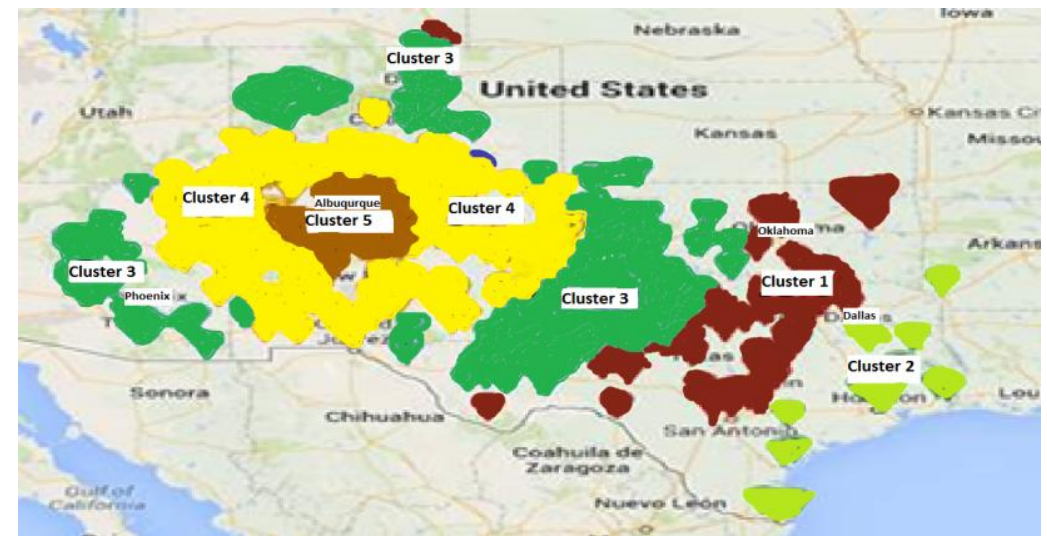

Fig1. The five clusters formed in the geographic area under consideration 
American Research Journal Of Medicine And Surgery, Volume 1, Issue 1, 2015

ISSN 2379-8955

Table 2. Average hourly arrival rates of patients for each cluster

\begin{tabular}{|c|c|}
\hline Cluster & Avg. Hourly Arrival Rate over 2 years \\
\hline Cluster 1 & 0.021759 \\
\hline Cluster 2 & 0.00370371 \\
\hline Cluster 3 & 0.20885417 \\
\hline Cluster 4 & 0.902835648 \\
\hline Cluster 5 & 3.23119213 \\
\hline
\end{tabular}

\section{Methodology}

The concept of reimbursing the qualified patients for their mileage specially if patients are required or forced to travel hundreds of miles to seek access to qualified healthcare staff as part of their provider network, increases the cost to the provider significantly. Additionally the patients bare the cost of mileage which is not reimbursed for whatever reason, along with the cost of meals while travelling away from home, and the cost of lodging when not admitted to the hospital or the medical center for either procedure(s) or surgery.

In many cases patients will be travelling with a companion who will still be required to pay for their own lodging while waiting on the patient to complete their surgery and recovery. This adds to the out-of-pocket cost to the patients and their family.

The simulation model is used to determine the cost of mileage reimbursement of the provider for any given year and the out-of-pocket cost to the patients. In cases that patient require surgery or a more complicated procedure, the patients are travelling to the medical center for pre-surgery screening to include all the testing and medical examinations. In all such cases, patients will also do follow up exams with their surgeons and/or their primary care provider which may require traveling to the medical center.All in all, the patients may travel a minimum of 2-3 times for a surgery and/or a more involved procedure in orthopedic and podiatry departments. Some of these visits might not be reimbursed, depending on the hospital's reimbursement policy.

If the cost to the provider calculated by the mileage reimbursed to the patients is high enough to support a rural facility to at least complete the pre and post-surgical visits, may result in decreased reimbursement dollars to the patients and more importantly, will significantly reduce the out-of-pocket cost to the patients and their families.

To test this hypothesis, we used simulation to generate entities/patients to travel to Albuquerque, NM from north, south and west of New Mexico as well as West Texas region. The patients' arrival was modeled after the actual arrival rate determined from the provider data using Arena Input Analyzer to determine the best fitting distribution. The cost model was also formulated based on the information provided by the provider on the reimbursement policy.

\section{Simulation MODEL}

Figure 2 shows the model developed using arena. Various calculations are done by placing modules, which are boxes of different shapes representing a series of processes or logics. Connector lines are used to connect the modules together. The connector represents the flow of entities. The modules act on the entities as specified or defined by the users and entities flow from one module or process to another as specified by the user. All output requirement set by the users are provided in a report format.

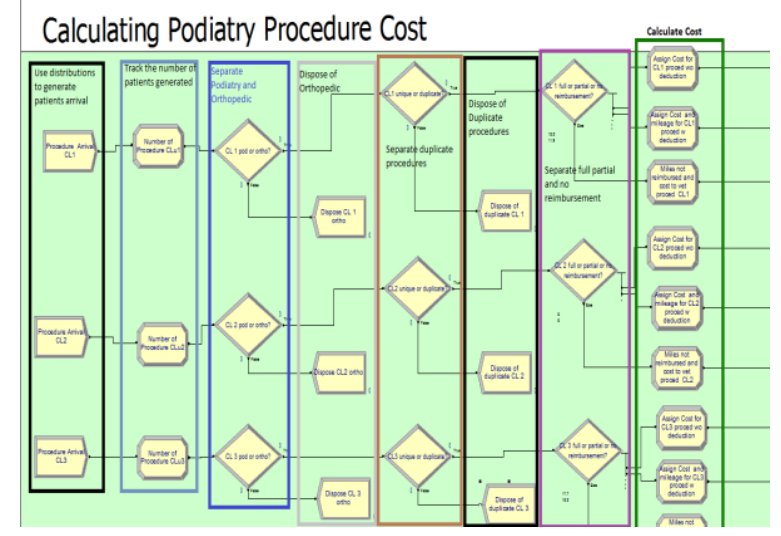

Fig2. The Model in ARENA 


\section{American Research Journal of Medicine and Surgery, Volume 1, Issue 1, 2015}

ISSN 2379-8955

The model is stochastic discrete event simulation and aims at calculating the cost associated with Podiatry procedures.The patients arrive according to a specified distribution(s) and the cost is calculated for each arrival according to the path which the entity takes. The parameters of the model were acquired by mining the data provided by the hospital. Patient's arrival distributions were generated for every month, resulting in 24 distributions for each cluster for the 2 year period. The data was mined for the percentage of podiatry patients within each cluster. The process of the cost calculation is shown in Figure 3. The arrival rate distributions were applied for each month independently by creating a schedule of arrival for each patient cluster for each month. The duplicate procedures were removed as they are not considered real visits; duplicate procedures occur when a patient undergoes more than one procedure during the same visit, hence he is not travelling any distance to perform those extra (duplicate) procedures. According to the data, some patients were fully reimbursed according to the miles they travelled, while some were partially reimbursed and others not reimbursed at all, all according to the hospital's policy. The patients who are partially reimbursed receive $\$ 6$ less than the fully reimbursed patients. Out of pocket cost was calculated for the patients who didn't get reimbursed. The out of pocket cost was calculated for the miles travelled and the lodging and meals cost. The average mileage cost is presented in Table 3.

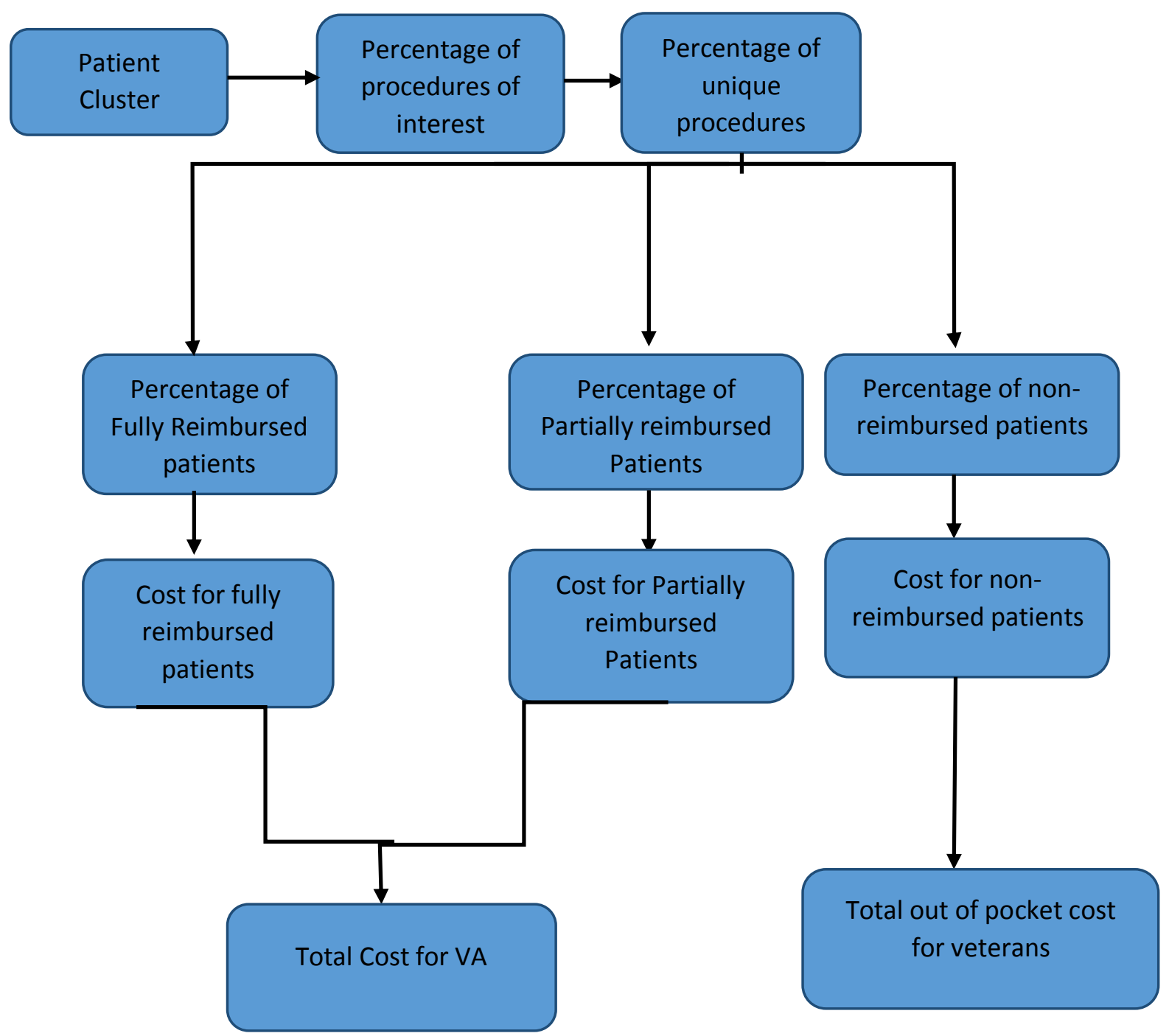

Fig3. Flow Chart of the Cost Calculation process 
American Research Journal Of Medicine And Surgery, Volume 1, Issue 1, 2015

ISSN 2379-8955

Table3. Average travel cost from each Cluster

\begin{tabular}{|l|l|}
\hline Cluster & Average Cost per patient \\
\hline CL1 & $\$ 507$ \\
\hline CL2 & $\$ 732$ \\
\hline CL3 & $\$ 344$ \\
\hline CL4 & $\$ 160$ \\
\hline CL5 & $\$ 20$ \\
\hline
\end{tabular}

\section{MODEL VERIFICATION}

The model was verified by comparing the total number of arrivals over the 2 years period obtained from the data and the simulation arrivals. Table 4 below shows that the distribution of the total number of arrivals over the various clusters mimics the behavior of the data's actual arrivals with a $5 \%$ difference in the total numbers of arrivals of all clusters.

Table4. Cluster arrival verification

\begin{tabular}{|c|c|c|c|}
\hline Patient Cluster & Simulation Arrivals & Actual Arrivals & Percentage Difference \\
\hline 1 & 424 & 377 & $12 \%$ \\
\hline 2 & 52 & 64 & $-19 \%$ \\
\hline 3 & 3,695 & 3,610 & $2 \%$ \\
\hline 4 & 14,152 & 15,601 & $9 \%$ \\
\hline 5 & 53,523 & 55,835 & $4 \%$ \\
\hline Total & 71,846 & 75,487 & $-5 \%$ \\
\hline
\end{tabular}

The other issue that was considered is the distribution of the patients' arrival over time. The rate of the arrival of patients varies over the year and it is important that the simulation model incorporates the different arrival rates over the 2 years period. Table 5 shows the total number of arrivals for all clusters combined over intervals of 2 month increments and compares them to the simulation arrivals. A Chi-Square Goodness of Fit test is performed with a Null Hypothesis that states that the distribution of arrivals in the data can be fitted with the distribution of arrivals in the simulation model.

Table5. Total arrivals over 2 month increments

\begin{tabular}{|l|l|l|l|l|}
\hline Time Period & $\begin{array}{l}\text { Simulation } \\
\text { Arrivals }\end{array}$ & \% of total for simulation & Actual Arrivals & $\begin{array}{l}\text { \% of total for } \\
\text { Actual }\end{array}$ \\
\hline 1-2 months & 6,304 & $8.4 \%$ & 6,327 & $8.77 \%$ \\
\hline 3-4 months & 6,111 & $8.2 \%$ & 6,217 & $8.51 \%$ \\
\hline 5-6 months & 5,987 & $8.4 \%$ & 6,313 & $8.33 \%$ \\
\hline 7-8 months & 6,055 & $9.0 \%$ & 6,796 & $8.43 \%$ \\
\hline 9-10 months & 5,900 & $7.7 \%$ & 5,800 & $8.21 \%$ \\
\hline $\mathbf{1 1 - 1 2}$ months & 6,940 & $6.0 \%$ & 4,510 & $9.66 \%$ \\
\hline $\mathbf{1 3 - 1 4}$ months & 4,074 & $9.3 \%$ & 7,009 & $5.67 \%$ \\
\hline $\mathbf{1 5 - 1 6}$ months & 5,583 & $8.3 \%$ & 6,273 & $7.77 \%$ \\
\hline $\mathbf{1 7 - 1 8}$ months & 5,982 & $8.3 \%$ & 6,264 & $8.33 \%$ \\
\hline $\mathbf{1 9 - 2 0}$ months & 6,336 & $9.3 \%$ & 7,018 & $8.82 \%$ \\
\hline $\mathbf{2 1 - 2 2}$ months & 6,504 & $9.0 \%$ & 6,803 & $9.05 \%$ \\
\hline $\mathbf{2 3 - 2 4}$ months & 6,070 & $8.0 \%$ & 6,327 & $8.45 \%$ \\
\hline Total & 71,846 & & & \\
\hline
\end{tabular}

Chi Square Goodness of Fit test

Null Hypothesis: The expected values of the simulation are a good fit of the observed values of the data

The FREQ Procedure 
American Research Journal of Medicine and Surgery, Volume 1, Issue 1, 2015 ISSN 2379-8955

\begin{tabular}{|r|r|r|r|r|r|}
\hline month & Frequency & Percent & $\begin{array}{r}\text { Test } \\
\text { Percent }\end{array}$ & $\begin{array}{r}\text { Cumulative } \\
\text { Frequency }\end{array}$ & $\begin{array}{r}\text { Cumulative } \\
\text { Percent }\end{array}$ \\
\hline $\mathbf{2}$ & 8.4 & 8.39 & 8.80 & 8.4 & 8.39 \\
\hline $\mathbf{4}$ & 8.2 & 8.19 & 8.50 & 16.6 & 16.58 \\
\hline $\mathbf{6}$ & 8.4 & 8.39 & 8.30 & 25 & 24.98 \\
\hline $\mathbf{8}$ & 9 & 8.99 & 8.40 & 34 & 33.97 \\
\hline $\mathbf{1 0}$ & 7.7 & 7.69 & 8.20 & 41.7 & 41.66 \\
\hline $\mathbf{1 2}$ & 6 & 5.99 & 9.70 & 47.7 & 47.65 \\
\hline $\mathbf{1 4}$ & 9.3 & 9.29 & 5.70 & 57 & 56.94 \\
\hline $\mathbf{1 6}$ & 8.3 & 8.29 & 7.80 & 65.3 & 65.23 \\
\hline $\mathbf{1 8}$ & 8.3 & 8.29 & 8.30 & 73.6 & 73.53 \\
\hline $\mathbf{2 0}$ & 9.3 & 9.29 & 8.80 & 82.9 & 82.82 \\
\hline $\mathbf{2 2}$ & 9.2 & 9.19 & 9.10 & 92.1 & 92.01 \\
\hline $\mathbf{2 4}$ & 8 & 7.99 & 8.40 & 100.1 & 100.00 \\
\hline
\end{tabular}

Chi-Square Test

for Specified Proportions

\begin{tabular}{|l|r|}
\hline Chi-Square & $\mathbf{3 . 8 6 5 0}$ \\
\hline DF & 11 \\
\hline Pr $>$ ChiSq & $\mathbf{0 . 9 7 3 7}$ \\
\hline
\end{tabular}

From the Chi Square test, since there is not enough evidence to reject the Null Hypothesis, we conclude the goodness of fit of the model to adequately represent the arrival behavior of the actual data.

\section{CONCLUSION}

Assuming that the following costs are used for mileage, meals, and lodging and that all patient travel to Albuquerque to seek care, the total cost associated with the provider and patients are calculated below.

- $\quad$ Mileage $-\$ 0.415 /$ miles

- $\quad$ Lodging - $\$ 83 /$ night

○ Travelling 401-799 (1 night of lodging)

- Traveling 800-1199 (2 nights of lodging)

○ Travelling 1200-1599 (3 nights of lodging)

- $\quad$ Meals - \$46/day

- A day is travelling 400 miles to destination

The simulation model generates patient arrivals based on the arrival rate distribution for each cluster independently. The reimbursement for the fully reimbursed patients was calculated as the average number of miles a patient travels from a given cluster multiplied by 2 to account for the round trip and finally multiplied by the reimbursement rate which is $\$ 0.415$ per mile. The cost associated with this model is shown in Table 6.

Table 6 show the mileage traveled and reimbursed by the provider for patients who were admitted for surgery and patients who completed an outpatient procedure. The total miles which were reimbursed by the provider in two year was $1,052,153$ miles which was equivalent to $\$ 436,643$ over 2 years. This amount seems large enough to warrant further investigation of the concept of rural clinic in order to minimize travel miles to reduce cost to the provider.

The cost to the patients was determined using the miles which were not reimbursed by the provider totaling $2,599,950$ miles for a total out-of-pocket miles cost to the patients of $\$ 1,078,979$. Adding the cost of meals and lodging for the patient only, assuming he travels alone, the total cost to the patient increases to $\$ 3,608,711$ over 2 years. 
This is a significant burden to the patients and their families and any effort to minimize this cost would benefit both the provider and their patients.

Table6. Cost breakdown for different type of patients and provider cost

\begin{tabular}{|l|l|}
\hline Simulation Results & $\mathbf{1 , 0 5 2 , 1 5 3}$ \\
\hline Miles Reimbursed by Provider & $\mathbf{2 , 5 9 9 , 9 5 0}$ \\
\hline Miles not Reimbursed by Provider & $\mathbf{\$ 4 3 6 , 6 4 3}$ \\
\hline Total Cost to Provider & $\mathbf{\$ 1 , 0 7 8 , 9 7 9}$ \\
\hline \hline Cost of Mileage to Patients & $\mathbf{\$ 2 , 5 2 9 , 7 3 2}$ \\
\hline Cost of Lodging \& Per Diem to Patients & $\mathbf{\$ 3 , 6 0 8 , 7 1 1}$ \\
\hline Total Cost To Patients &
\end{tabular}

\section{ACKNOWLEDGEMENT}

Funding for this project was provided by the Department of Veterans Affairs; Veterans Health Administration; Midwest Mountain Veterans Engineering Resource Center (MWM VERC). The views expressed in this presentation are those of the authors and do not necessarily reflect the position or policy of the United States government or any of its agencies. The authors have no conflict of interest to disclose.

\section{REFERENCES}

[1] Bowers, J. A. (2011). Simulating waiting list management. Health care management science, 14(3), 292-298.

[2] Creemers, S., Lambrecht, M., \& Vandaele, N. (2007). Queueing models in healthcare. Tijdschrift voor Economie en Management, 52(3), 471.

[3] Duguay, C., \& Chetouane, F. (2007). Modeling and improving emergency department systems using discrete event simulation. Simulation, 83(4), 311-320.

[4] El- Darzi, E., Vasilakis, C., Chaussalet, T., \& Millard, P. H. (1998). A simulation modelling approach to evaluating length of stay, occupancy, emptiness and bed blocking in a hospital geriatric department. Health Care Management Science, 1(2), $143-149$

[5] Knottnerus, J. A., Joosten, J. O. L. A. N. D. A., \& Daams, J. (1990). Comparing the quality of referrals of general practitioners with high and average referral rates: an independent panel review. The British Journal of General Practice, 40(334), 178.

[6] Meijboom, B., Schmidt-Bakx, S., \& Westert, G. (2011). Supply chain management practices for improving patient-oriented care. Supply Chain Management: An International Journal, 16(3), 166-175.

[7] Niu, Q., Peng, Q., El Mekkawy, T., Tan, Y. Y., Bruant, H., \& Bernaerdt, L. (2011). Performance analysis of the operating room using simulation. Proceedings of the Canadian Engineering Education Association.

[8] Peltokorpi, A., Lehtonen, J. M., Torkki, P., \& Moilanen, T. Optimal queue length for orthopaedic surgery with surgeonspecific queues and maximum waiting time. In Proceedings of the 35th International Conference on Operational Research Applied to Health Services ORAHS, July 12-17, 2009, Leuven, Belgium.

[9] Schofield, W. N., Rubin, G. L., Piza, M., Lai, Y. Y., Sindhusake, D., Fearnside, M. R., \& Klineberg, P. L. (2005). Cancellation of operations on the day of intended surgery at a major Australian referral hospital. Med J Aust, 182(12), 612615.

[10] VanBerkel, P. T., \& Blake, J. T. (2007). A comprehensive simulation for wait time reduction and capacity planning applied in general surgery. Health care management Science, 10(4), 373-385.

[11] Yang, Y., Sullivan, K. M., Wang, P. P., \& Naidu, K. D. (2000, February). Applications of computer simulation in medical scheduling. In Proceedings of the joint conference on information sciences (Vol. 227)

\section{AUTHORS' BIOGRAPHY}

Kambiz Farahmand is currently a professor at the Industrial and Manufacturing Engineering and Management at North Dakota State University. He is an internationally recognized expert in Productivity Improvement. Dr. Farahmand has over 30 years of experience as an engineer, manager, and educator. His primary teaching and research activities are in the areas of Productivity Improvement of Manufacturing Systems, Human Exposure and Physiology Simulation, Simulation and Modeling, Operations \& Materials Management and Strategic Planning, and Healthcare Management. He is a registered professional engineer in the states of Texas and North Dakota.

Sudhindra Upadhyaya is currently a PhD student in the department of Industrial and Manufacturing Engineering at North Dakota State University. 
Vahid H Khiabani is currently an assistant professor of Operations Management at Minnesota State University Moorhead. He received his PhD in Industrial Engineering from North Dakota State University in 2014. His research interests include reliability modeling and optimization of smart grid, healthcare process improvement, and mathematical and probabilistic modeling.

Mahmoud Mostafa is currently a Masters student in the department of Industrial and Manufacturing Engineering at North Dakota State University.

Satpal Singh Wadhwa is currently a PhD student in the department of Industrial and Manufacturing Engineering at North Dakota State University. 